\title{
Basics of Colour Science and Prosthodontics - A Review
}

\section{Aswathy S Kumar ${ }^{1}$, Lekshmy AR ${ }^{1}$, Pradeep Dathan ${ }^{2}$ and K Chandrasekharan Nair ${ }^{3 *}$}

${ }^{1}$ Postgraduate Student, Department of Prosthodontics, Sri Sankara Dental College, Akathumuri, Thiruvavananthapuram, Kerala, India

${ }^{2}$ Professor and Head of the Department of Prosthodontics, Sri Sankara Dental

College, Akathumuri, Thiruvavananthapuram, Kerala, India

${ }^{3}$ Professor Emeritus, Department of Prosthodontics, Sri Sankara Dental College, Akathumuri, Thiruvananthapuram, Kerala, India

*Corresponding Author: K Chandrasekharan Nair, Professor Emeritus, Department of Prosthodontics, Sri Sankara Dental College, Akathumuri, Thiruvananthapuram, Kerala, India.
Received: October 05, 2021

Published: October 28, 2021

(C) All rights are reserved by

K Chandrasekharan Nair., et al.

\section{Abstract}

Colour is an important factor that dictates the success of clinical practice in Prosthodontics. The science behind colour has to be studied in depth to have an understanding of the modern developments in gadgetry related to shade selection. This review is an attempt to throw light on the basics of colour science related to Prosthodontics.

Keywords: Teeth; Colour; Shade; Shade Guide; Shade Match; Colour Space; Spectrophotometers; Munsell Colour System

\section{Introduction}

Advancements in technology, computers, Internet and communication systems have jointly and independently shaped the modern society. Commensurate with these strides, much advancement in contemporary dentistry has happened. During the past decade, dental profession has experienced the growth of a new generation of technologies devoted to the analysis, communication and verification of shade. Shade determination for direct and indirect restorations has always been a challenge for the Prosthodontist. For many dental patients, restoring dental esthetics is of high importance in the course of their dental treatment. Blending of restorative materials with the teeth and producing natural appearance should be the major goal of the restorative dentist.
The wavelengths present in a beam of visible light determine the property which is referred to as color. Visible light is a form of electromagnetic energy with the wavelength range of 400-700 nm which can easily be detected by the human eye. An object becomes visible when it emits light, or when it reflects or transmits incident light from an external source. It can be said that the perception of color is the result of a physiological response to a physical stimulus.

\section{Structure of the eye}

Light from an object that falls on the eye is focused onto the retina by the lens. The light is then converted into nerve impulses and subsequently gets transmitted to the cerebral cortex. The retina is made up of rod and cone-shaped cells. The cone shaped cells are re- 
sponsible for the color vision which is estimated to be six to seven million. The cones are functionally divided into "red" cones, "green" cones, and "blue" cones. In contrast, the rod cells are monochromatic and are efficient photo receptors. They are responsible for the dark-adapted vision. The number of rods is estimated to be 120 million in a human being.

An individual can perceive color as a result of physiological response to a physical stimulus. Human beings can perceive colour differences but may have subjective variations.

Colorimeters and spectrophotometers can also process reflected light emerging from the surface of an object and can calculate the light flux data into color parameters that can be used to determine shade and calculate color differences (Figure 1).

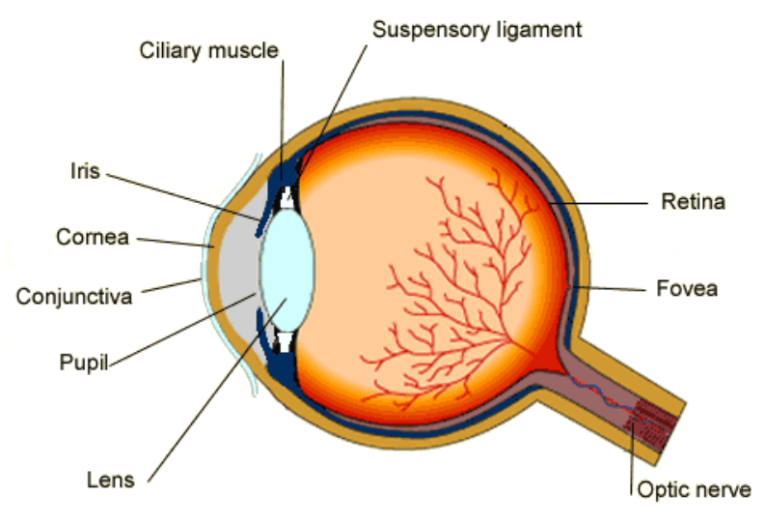

Figure 1: Structure of the eye. (https://socratic.org/questions/ what-are-the-functions-of-the-cornea-pupil-lens-retina-and-opticnerve).

\section{Colour vision deficiency [1]}

Dental professionals who are interested in aesthetic restorative dentistry should have a high level of colour distinguishing ability. Hence they have to get their colour vision screened periodically. For that purpose, Ishihara's test for color deficiency is popularly used. This test contains 38 plates of circles created by irregularly coloured dots in two or more colours. The plates will be put in front of a person and will be asked to identify the number which can be seen on each plate. If certain number of errors is made, it should raise the doubt that the person is colour blind.

According to world statistics, colour blindness (colour vision deficiency, or CVD) affects approximately 1 in 12 men and 1 in 200 women in the world. It is estimated that there are approximately 300 million people with colour blindness all over the world. Colour blindness is predominantly a genetic (hereditary) condition. Red/ green and blue colour blindness is inherited from the parents. The gene causing the colour blindness is carried through the $\mathrm{X}$ chromosome and that is why more men are affected than women. Chronic illnesses like diabetes mellitus, glaucoma, leukemia, liver diseases, chronic alcoholism, macular degeneration etc. can cause acquired colour vision defects. Medications such as antibiotics, barbiturates, anti-tuberculosis drugs and anti hypertensives can also cause colour blindness. Advancing age, accidents that damage the retina and industrial chemicals like carbon monoxide are also the reasons for acquired colour blindness. About $2 \%$ of the male population is red-green color blind because of a lack of the red or green pigment (conditions called protanopia and deuteranopia, respectively). Red-green colour blind people have difficulty in distinguishing between red, green, brown and orange (Figure 2,3).

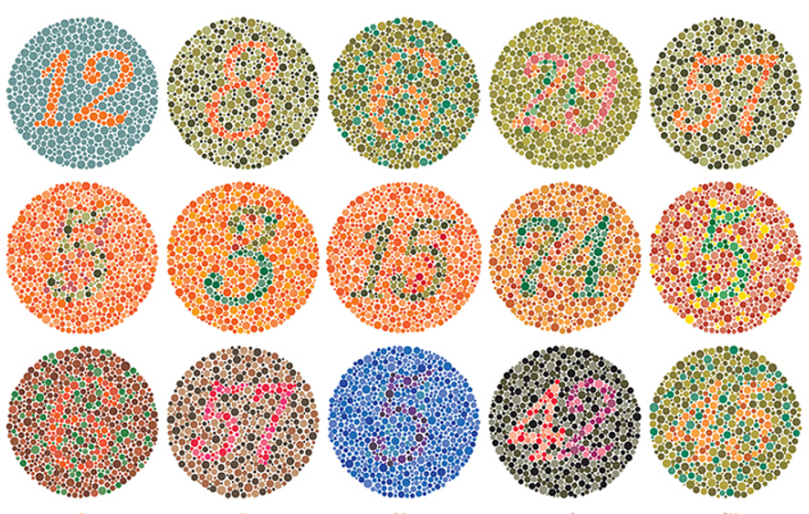

Figure 2: Ishihara's colour vision test. (https://www.aoa. org/healthy-eyes/eye-and-vision-conditions/color-visiondeficiency?sso=y). 


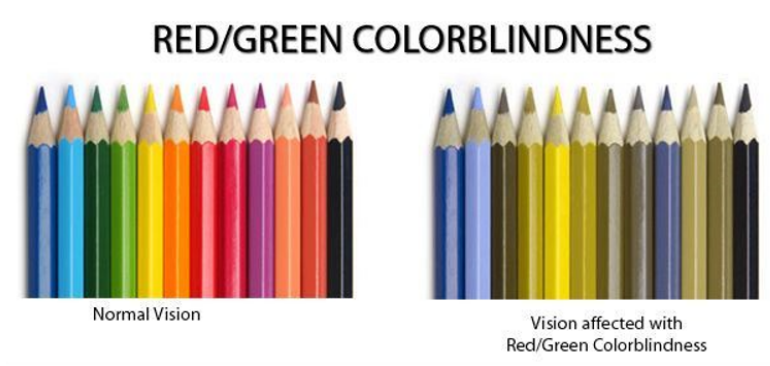

Figure 3: Effect of colour blindness. (http://myomini.blogspot. com/2018/10/color-blindness.html).

\section{Munsell color system}

Munsell three-dimensional color space was created by Professor Albert H. Munsell in the first decade of the $20^{\text {th }}$ century. There are three attributes for this system viz. Hue (H), Chroma (C), and Value (V), and they are written in the form $\mathrm{H} \mathrm{V/C}$, which is called the Munsell notation. These three dimensions are also known as tristimulus data-perceptual attributes of color that allows the brain to interpret and conceptualize the signals it receives through visual stimuli. Hue is the name of color as observed in the spectrum, which is divided into five major groups: Red, Yellow, Green, Blue, and Purple, along with five intermediate hues halfway between adjacent principal hues (Figure). Hue is measured by degrees around horizontal circles. Chroma is measured radially outward from the neutral (gray) vertical axis and indicates the purity of the colour. Value is the lightness or darkness of a color varied vertically on the core cylinder from black (value 0) at the bottom, to white (value $10)$ at the top. Between black and white, neutral grays are placed along the vertical axis. The Munsell color system was the first system to separate hue, value, and chroma into independent dimensions in a three dimensional space. Munsell determined the spacing of colors based on human visual responses (Figure 4) [2].

\section{CIE Lab color space}

Human eye is capable of seeing many colors but it will not always distinguish colors from one another accurately. Slightly dif-

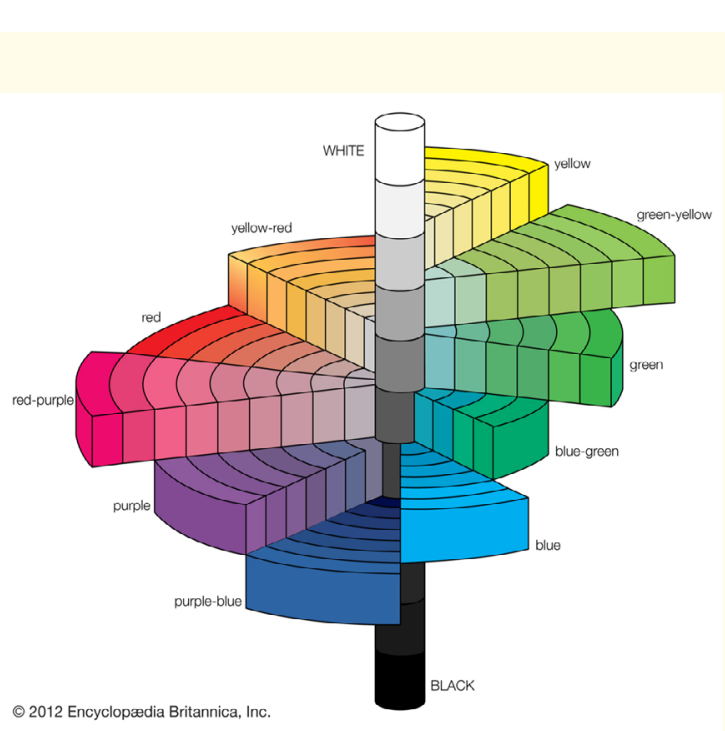

Figure 4: Munsell Colour System. (https://www.britannica.com/ science/Munsell-color-system).

ferent colors may be perceived as the same or see differences in objects of identical color depending on the viewing angle and lighting. This creates challenges in communicating about color. The International Commission on Illumination (CIE) developed the $\mathrm{L}^{*} \mathrm{a}^{*} \mathrm{~b}^{*}$ color model in 1976 with the intent of creating a standard for color communication. CIE developed CIELAB to simplify color communication regardless of the device used. The color space is copyright- and license-free and it can be integrated into any device or system. The CIE in CIELAB is the abbreviation for the International Commission on Illumination's French name, Commission Internationale de l'Eclairage. The letters $\mathrm{L}^{*}, \mathrm{a}^{*}$ and $\mathrm{b}^{*}$ represent each of the three values, the CIELAB color space uses to measure objective color and calculate color differences. $\mathrm{L}^{*}$ represents lightness from black to white on a scale of zero to 100 , while $a^{*}$ and $b^{*}$ represent chromaticity with no specific numeric limits. Negative $a^{*}$ corresponds with green, positive $\mathrm{a}^{*}$ corresponds with red, negative $b^{*}$ corresponds with blue and positive $b^{*}$ corresponds with yellow (Figure 5). Before performing any $\mathrm{L}^{*} \mathrm{a}^{*} \mathrm{~b}^{*}$ calculations, the sample's $\mathrm{L}^{*}, \mathrm{a}^{*}$ and $\mathrm{b}^{*}$ values has to be determined. To do this, spectrophotometry instrumentation has to be done. 


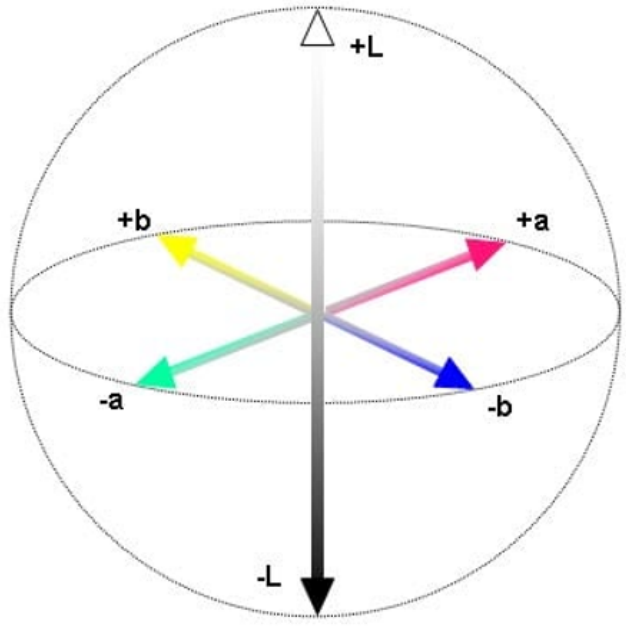

Figure 5: CIE Lab colour space. (https://www.researchgate.net/ publication/338303610_Research_Techniques_Made_Simple).

\section{Quantifying color difference $(\Delta)$}

The delta values $\Delta \mathrm{L}^{*}, \Delta \mathrm{a}^{*}$, and $\Delta \mathrm{b}^{*}$ indicate how much a standard and sample differ fromone another in $\mathrm{L}^{*}, \mathrm{a}^{*}$, and $\mathrm{b}^{*}$. The total color difference $\Delta \mathrm{E}$ is calculated using the following equation (Figure 6).

$$
\Delta E_{a b}^{*}=\sqrt{\Delta L^{* 2}+\Delta a^{*^{2}}+\Delta b^{*^{2}}}
$$

Figure 6: Equation used to calculate Delta E.

There are other color difference formulae in use today; however the CIELAB $\triangle \mathrm{E}$ ab is still the most widely used in color research in dentistry. This is a single value which takes into account the differences between the $L^{*}, a^{*}$, and $b^{*}$ of the sample and standard. Under controlled conditions, $\Delta \mathrm{E}$ value of 1 or greater can be perceived by the human eye. The smallest difference in color detected by human observers is $\Delta \mathrm{E}$ value of 1 . However, under clinical conditions, $\Delta \mathrm{E}$ of 3.3 has been shown to be the upper limit for human eyes to detect color differences. The $\Delta \mathrm{E}$ value greater than 3.7 indicates a poor match and colour difference can be observed easily. Intra orally, minor differences between shade tab and teeth appear to be matched because of the background distractions such as the mucosa, the gingiva, and shadowing caused by the lips.

\section{Shade guides [2-4]}

Dental shade-matching instruments are used to reduce imperfections and inconsistencies of traditional shade matching. Shade matching is commonly done by visual method. Vitapan Classical (Vita Zahnfabrik, Germany) and its derivations are probably the most commonly used shade guides. Introduction of evidence-based Vitapan 3D-Master shade guides, Tooth guide, Bleached guide and particularly Linear guide correspond to color of human teeth and optimized successful shade matching.

\section{VITA classical}

The VITA Classical A1-D4 shade guide was introduced in 1956 (Figure 7). The VITA Classical A1-D4 consists of sixteen tabs. The tabs are arranged into four groups (A-D) based on hue with increasing chroma within the groups as follows:

- A1 - A4 (reddish-brown)

- B1 - B4 (reddish-yellow)

- $\quad$ C1 - C4 (greyish shades)

- $\quad$ D2 - D4 (reddish-grey)

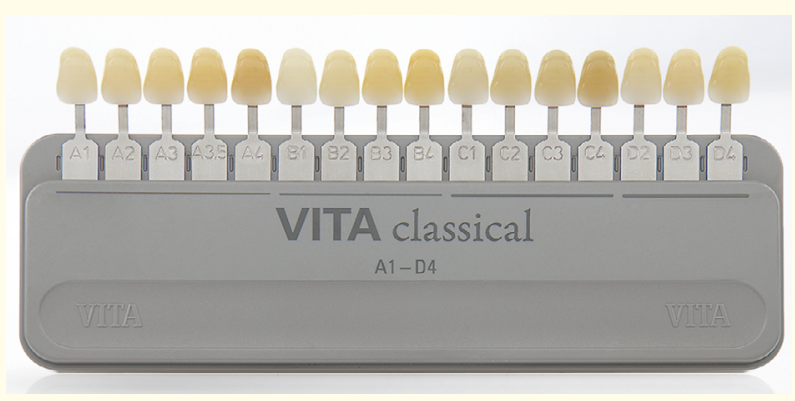

Figure 7: Vita Classical-A1-D4 shade guide. (https://www. dentaltix.com/en/blog/how-use-vita-colour-guide-find-best-dentalcomposite). 
However, the tabs can be rearranged according to value and some dentists prefer that for clinical use. For that the tabs are arranged as follows: B1, A1, B2, D2, A2, C1, C2, D4, A3, D3, B3, A3,5, B4, C3, A4, C4 (Figure 8).

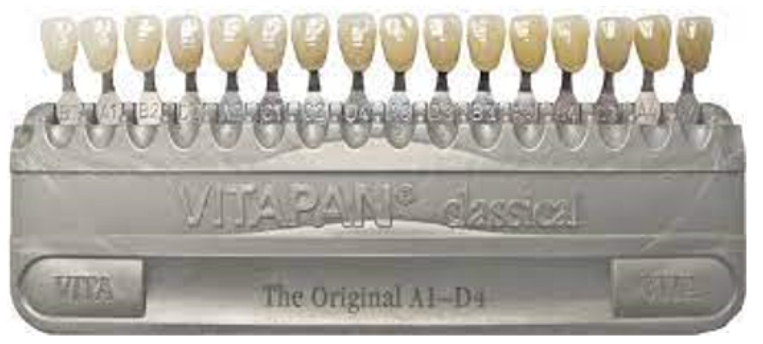

Figure 8: Vita classical arranged according to value.

\section{D Master}

The 3D master shade guide consists of 26 tabs arranged in five lightness (value) groups, with the tabs in each group has approximately the same lightness (value). The $\Delta \mathrm{E}$ value difference between the groups is approximately set at 4 which is easily recognized by most human eyes. Within the lightness group, the tabs are arranged in five different chromas $(1,1.5,2,2.5$ and 3), again with approximately the same $\Delta \mathrm{E}$ difference. Within the group three hues are arranged (L,M,R). L indicates yellowish, $\mathrm{R}$ - reddish and $\mathrm{M}$ - neutral. Within each group, the tabs are arranged vertically according to chroma and horizontally according to hue (Figure 9).

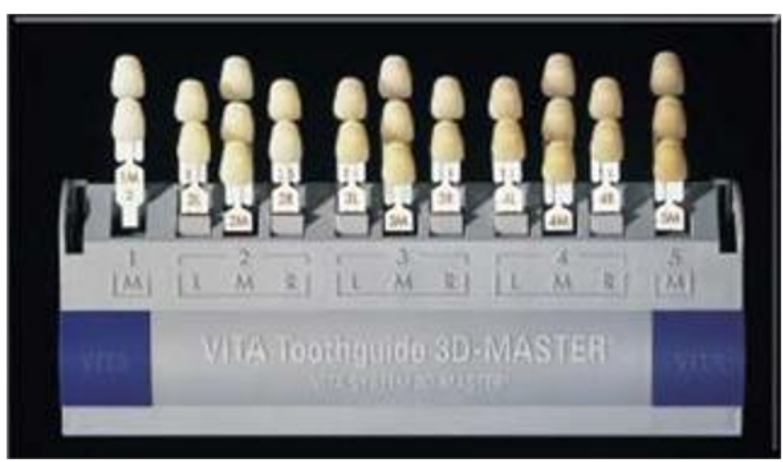

Figure 9: 3D Master shade guide.
The shade-matching process is done in three steps: 1. selection of the lightness (value) group from the five tabs (1-5) of lowest chroma (1) and neutral hue (M). Chroma is then determined with the middle three tabs of the respective lightness group and, finally, the hue group (L/M/R). With 3D master, fewer mismatches do occur than with the Vita Classical.

\section{Vita 3D linear guide (LG) [5]}

LG consists of six linear shade guides of which one is a value guide and five are chroma/hue guides, each guide having three to seven tabs. The tooth colour matching is done in two phases. First, the lightness (Value) is selected using the value guide with middle chroma and neutral hue. Each value tab has a corresponding chroma/hue guide. From that correct chroma and hue can be selected (Figure 10).

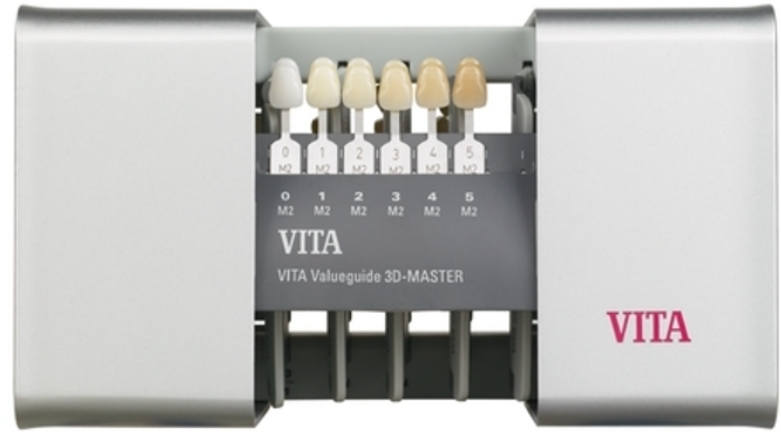

Figure 10: 3D-master-linear-shade-guide.

Visual assessment of shade has several innate difficulties: viz. metamerism (a phenomenon that occurs when two colors appear to match under one lighting condition, but not when the light changes), suboptimal color matching conditions, the evaluator's age, fatigue, mood and the drugs used. In spite of these difficulties, the human eye can distinguish minute differences in color. Some operators use to squint the eye while selecting the shade because it reduces the light, diminishes cone sensitivity and enhances rod sensitivity. Value selection becomes more accurate because rods are responsible for that. However, the ability to communicate the degree and nature of these differences is not without limitations. 


\section{Stump shade guide [6]}

The final color of an all-ceramic restoration is a merging of that of underlying tooth structure or core and the ceramic material. The color of the final restoration cannot match the shade selected from a shade guide unless this modification is taken into account. Therefore, a stump or shade of the prepared tooth needs to be assessed and the technician has to be informed. Ivoclar Vivadent Natural Die Material Shade Guide is commonly used for this purpose. It includes nine different shades varying from bleached to extremely discolored preparations. Lab technician can use this information to make a die that has a shade similar to the tooth preparation. Hence the technician can make sure that there is no show-through or shade change to the ceramic from the underlying tooth structure (Figure 11).

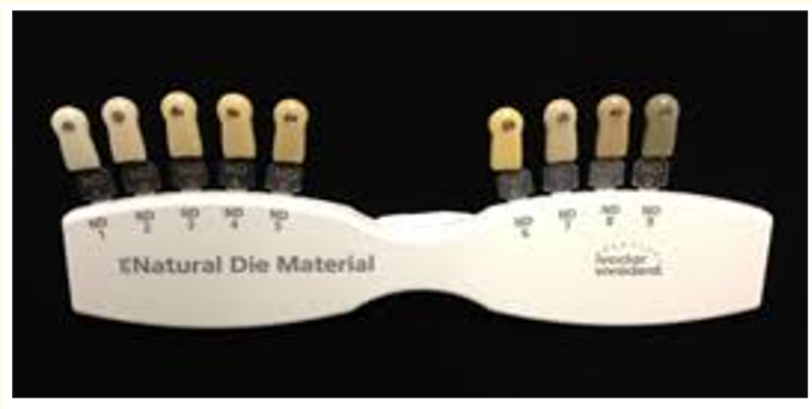

Figure 11: Stump shade guide.

\section{Spectrophotometers [7]}

Spectrophotometers are very accurate instruments used for color matching and they are adapted to dentistry too. A spectrophotometer measures the spectral reflectance or transmittance curve of an object. Light is released from a light source in the spectrophotometer and then dispersed through a prism into a spectrum of different wavelengths between $380-780 \mathrm{~nm}$. The spectrophotometer then measures the quantity of the light reflected from the specimen for each wavelength in the visible light, and converts the data into numerical values of colour coordinates - CIE L*, a* and b. Compared with observations by the human eye, spectrophotometers give nearly $33 \%$ increase in accuracy.

Vita Easyshade Compact (Vita Zahnfabrik, Germany) is cordless, small, portable, battery operated, contact-type spectrophotometer that provides adequate information on shade (Figure). Different measurement modes are possible with Easyshade Compact: tooth single mode, tooth area mode (cervical, middle and incisal shades), restoration color verification (includes lightness, chroma and hue comparison) and shade tab mode (practice/training mode). Shade matching has tread into a new dimension with the arrival of advanced instruments. Not only in clinical applications, are these instruments increasingly used in research also.

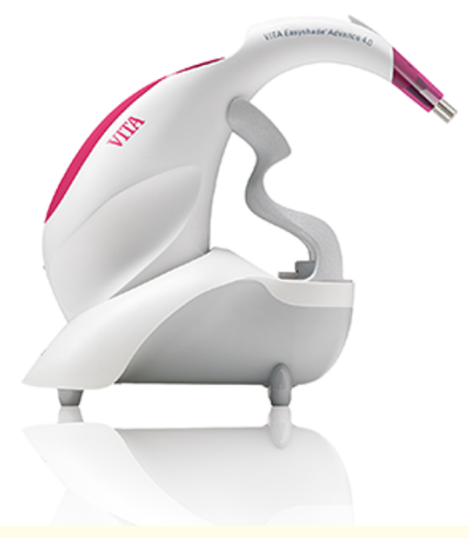

Figure 12: Vita Easy Shade Compact.

\section{Conclusion}

Assessment of tooth shade is essential for the clinical success of aesthetic restorations. Restorations should match with natural teeth or with other restored teeth. Visual judgment of the operators and patients form the basis of aesthetic assessment. Shade guides like VITA Classical, 3D Master, Linear Guide and Stump guide are in use for a long time. Operators should get their colour vision tested periodically because advancing age, diseases and many drugs can cause colour vision disability. Use of Spectrophotometers is at present gaining popularity because of the accuracy. May be in future Artificial Intelligence may take over the shade selection process completely.

\section{Bibliography}

1. Ragain J C. "A Review of Color Science in Dentistry". Journal of Dentistry, Oral Disorders and Therapy 3.1 (2015): 1-4. 
2. Jiun-Yao Chang., et al. "Evaluating the accuracy of tooth color measurement by combining the Munsell color system and dental Colorimeter". Kaohsiung Journal of Medical Sciences 28 (2012): 490-494.

3. Hassel A J., et al. "Clinical effect of different shade guide systems on the tooth shades of ceramic veneered restorations". International Journal of Prosthodontics 18 (2005): 422-426.

4. Stephen J Chu., et al. "Dental color matching instruments and systems. Review of clinical and research aspects". Journal of Dentistry 38 (2010): e2-e16.

5. Corcodel., et al. "The linear shade guide design of Vita 3Dmaster performs as well as the original design of the Vita 3Dmaster". Journal of Oral Rehabilitation 37 (2010): 860-865.

6. Luke S Kahng. "Hidden effects of stump shades". Dental Lab Products, February (2010).

7. N ALGhazali., et al. "Performance Assessment of Vita Easy Shade Spectrophotometer on Colour Measurement of Aesthetic Dental Materials". European Journal of Prosthodontics and Restorative Dentistry 19.4 (2011): 168-174.

Volume 5 Issue 11 November 2021

(C) All rights are reserved by $\mathrm{K}$ Chandrasekharan Nair., et al. 